\title{
Ação educativa acerca dos fatores de riscos de doenças cardiovasculares em adolescentes: relato de experiência
}

Educational action on cardiovascular disease risk factors in adolescents: experience report

Acción educativa sobre factores de riesgo de enfermedad cardiovascular en adolescentes: informe de experiencia

Amanda Rodrigues Figueiredo ${ }^{1 *}$, Ana Beatriz Souza Cabral ${ }^{1}$, Ana Caroline Oliveira Almeida ${ }^{1}$, Márcio Alves Ribeiro ${ }^{1}$, Maria Rute de Souza Araújo ${ }^{1}$, Milena Farah Damous Castanho Ferreira ${ }^{1}$, Adriano Heleno Costa da Silva².

\section{RESUMO}

Objetivo: Relatar uma ação educativa acerca dos fatores de risco de doenças cardiovasculares em adolescentes em uma escola no município de Belém. Relato de experiência: $A$ ação foi dividida em três momentos, no primeiro foi realizado uma roda de conversa sobre o estilo de vida dos participantes e histórico familiar. No segundo momento foi realizada uma explanação oral, com auxilio do método criado pelas autoras sobre como ocorre o processo fisiopatológico da aterosclerose. Por fim, no terceiro momento foi realizada uma dinâmica sobre o tema para verificar a sedimentação do aprendizado. Participaram da ação 18 alunos do ensino fundamental, sendo 11 meninas (61\%) e 7 meninos (39\%), com faixa etária de 12 a 14 anos. Constatou-se que a maioria dos alunos não era praticante de qualquer atividade física. Considerações finais: Considerando a experiência prática vivenciada nesta ação educativa as autoras observaram em seus achados que os adolescentes adotaram atualmente um estilo de vida de acordo com seu dia a dia e estão vivendo com hábitos irregulares e comportamentos sedentários que compõem de forma natural a sua rotina social e familiar, o que pode ocasionar em DCV precoce.

Palavras-Chave: Doenças cardiovasculares, Fatores de risco, Educação em saúde.

\section{ABSTRACT}

Objective: Report an educational action on the risk factors of cardiovascular disease in adolescents in a school in the city of Belém. Experience report: The action was divided into three moments, the first was a conversation about the lifestyle of adolescents. Participants and family history. In the second moment, an oral explanation was performed, with the help of the method created by the authors on how the pathophysiological process of atherosclerosis occurs. Finally, in the third moment there was a dynamic on the theme to verify the sedimentation of learning. Eighteen elementary students participated in the action, 11 girls $(61 \%)$ and 7 boys $(39 \%)$, aged 12 to 14 years. It was found that most students were not engaged in any physical activity. Final considerations: Considering the practical experience lived in this educational action, the authors observed in their findings that adolescents currently adopt a lifestyle according to their daily lives and are living with irregular habits and sedentary behaviors that naturally make up their social and family routine, which can lead to early CVD.

Key words: Cardiovascular disease, Risk factors, Educational action.

${ }^{1}$ Centro Universitário Metropolitano da Amazônia (UNIFAMAZ), Belém - PA.

*E-mail: amandar.figueiredo29@gmail.com

¿Universidade da Amazônia (UNAMA), Belém - PA.

SUBMETIDO EM: 11/2019

ACEITO EM: 12/2019

PUBLICADO EM: 2/2020

REAS/EJCH | Vol.Sup.n.42 | e2292 | DOI: https://doi.org/10.25248/reas.e2292.2020 Página 1 de 6 


\section{RESUMEN}

Objetivo: Informar una acción educativa sobre los factores de riesgo de enfermedad cardiovascular en adolescentes en una escuela de la ciudad de Belém. Informe de experiencia: La acción se dividió en tres momentos, el primero fue una conversación sobre el estilo de vida de los adolescentes. Participantes e historia familiar. En el segundo momento, se realizó una explicación oral, con la ayuda del método creado por los autores sobre cómo se produce el proceso fisiopatológico de la aterosclerosis. Finalmente, en el tercer momento hubo una dinámica sobre el tema para verificar la sedimentación del aprendizaje. Dieciocho estudiantes de primaria participaron en la acción, 11 niñas (61\%) y 7 niños (39\%), de 12 a 14 años. Se descubrió que la mayoría de los estudiantes no realizaban ninguna actividad física. Consideraciones finales: Teniendo en cuenta la experiencia práctica vivida en esta acción educativa, los autores observaron en sus hallazgos que los adolescentes actualmente adoptan un estilo de vida de acuerdo con su vida cotidiana y viven con hábitos irregulares y comportamientos sedentarios que naturalmente forman su rutina social y familiar, lo que puede conducir a una ECV temprana.

Palabras clave: Enfermedad cardiovascular, Acción educativa, Factores de riesgo.

\section{INTRODUÇÃO}

Doenças Cardiovasculares (DCV) correspondem a um grupo de doenças relacionadas a danos no coração e nas redes arteriais e venosas, com ampla variedade, graus de acometimento e risco a vida. Está no rol das doenças crônico-degenerativas não transmissíveis, por não terem sua origem ligada diretamente à ação e disseminação de agentes microbiológicos e, sim, a fatores de risco diversos, os quais geram propensão ao desenvolvimento dessas patologias. Esses fatores de risco podem ser diferenciados em dois tipos: os modificáveis (evitáveis) e não modificáveis (inevitáveis) (OPAS, 2017).

Dentre os fatores de risco modificáveis, estão em maior parte os hábitos de vida não saudáveis, destacando-se o tabagismo, o etilismo demasiado, as dislipidemias, o diabetes mellitus (DM), o sobrepeso, a obesidade, o sedentarismo, a hipertensão arterial sistêmica (HAS), o estresse, a má qualidade do sono e o uso de determinados medicamentos, a exemplo dos anti-inflamatórios não esteroidais (AINEs). Os fatores não modificáveis se relacionam com a hereditariedade, o sexo e a idade, entre outros (VALE MGE, et al., 2018).

Cinco fatores de risco para essas enfermidades estão relacionados com as condições de vida e estima-se que sejam os responsáveis por aproximadamente $40 \%$ da mortalidade em todo o mundo, entre eles estão: hipertensão arterial sistêmica (HAS) (13\%), tabagismo (9\%), hiperglicemia (6\%), sedentarismo (6\%), sobrepeso e obesidade (5\%) (AUDI CAF, et al., 2016).

O processo aterosclerótico pode se iniciar desde a infância, e a sua gravidade é proporcional ao número de fatores de riscos agregados, que se avança conforme o com o avanço da idade. $O$ estilo de vida de crianças e adolescentes, em especial as que apresentam histórico familiar positivo para doenças coronarianas, sedentarismo, sobrepeso e mantém ingestão excessiva de alimentos ricos em gorduras e açúcares, é determinante para que estes fatores de riscos influenciem no surgimento precoce de DCV. (SANTOS EGR, et al., 2019; FONTES VS, et al., 2018).

Calcula-se que 7,4 milhões de óbitos ocorrem devido às doenças cardiovasculares, que constituem três quartos (75\%) das mortes e 6,7 milhões devido a acidentes vasculares cerebrais (AVC). Destaca-se que 17 milhões dos óbitos ocorreram em pessoas com idade inferior a 70 anos por doenças crônicas não transmissíveis, $37 \%$ referem-se às doenças cardiovasculares, destes $82 \%$ dos casos em países de baixa e média renda (VALE MEG, et al., 2018; OPAS, 2017). O adoecimento vascular ocorre por diversos fatores e tem quase sempre uma detecção tardia, na maioria dos casos, devido ao não surgimento de sintomas mais evidentes no início da doença e ao índice de mortalidade ser mais proeminente na vida adulta. De fato, essas doenças se desenvolvem ao longo da vida, de forma lenta e gradual, tendo a infância como seu ponto de deflagração (FARIAS AM, et al., 2016). 
A ideia errônea de que os fatores de risco cardiovasculares e as DCV estejam presentes apenas em fases da vida mais avançadas colabora para a existência destes em fases da infância e adolescência, com evidências de aterosclerose já na idade jovem. Questões importantes neste período da vida, como a busca de estabilidade nas relações pessoais, aceitações interpessoais, dentre outros, tornam os adolescentes vulneráveis ao consumismo contemporâneo, que, com sua influência, interfere nos comportamentos da saúde (FONTES VS, et al., 2018).

Vale frisar a necessidade e importância de ações educativas para a promoção da saúde a fim de prevenir possíveis patologias relacionadas ao estilo de vida de crianças e adolescentes. A escola, por ser um ambiente de aprendizado e convívio social, é fundamental na elaboração de ações educativas por meio, principalmente, de práticas de atividades físicas, incentivo e informação sobre os seus benefícios (LIMA KLL, et al., 2015).

Dessa forma, o objetivo geral desse ensaio é relatar uma experiência através de uma ação educativa acerca dos fatores de risco de doenças cardiovasculares em adolescentes em uma escola de ensino fundamental e médio no município de Belém do Pará.

\section{RELATO DE EXPERIÊNCIA}

Tratou-se de um relato de experiência sobre uma ação educativa abordando os fatores de risco das doenças cardiovasculares em adolescentes em uma escola no município de Belém do Pará. A ação educativa foi desenvolvida na Escola de Ensino Fundamental e Médio Disneylândia localizada no Conjunto Jardim Maguari, no município de Belém no Estado do Pará. Ocorreu dia 29 de outubro de 2019 no horário entre 10 e 11 horas da manhã. O público alvo foi uma turma composta por 18 alunos do $8^{\circ}$ ano do ensino fundamental.

A ação ocorreu em três momentos. No primeiro, foi realizada uma roda de conversa sobre o estilo de vida dos participantes e seus históricos familiares para doenças cardíacas e também se estabeleceu uma reflexão sobre os impactos do sedentarismo, má alimentação e o desenvolvimento de doenças cardiovasculares. No segundo momento foi realizada uma explanação sobre o processo fisiopatológico da aterosclerose. Foi utilizado como recurso didático um equipamento construído para reproduzir o sistema cardiovascular, com circulação sanguínea e acometido por placas de ateroma.

A confecção do instrumento incluiu um painel amadeirado, mangueiras, placa de EVA, folha de EPS (Isopor), silicone em gel, água corada em vermelho e bomba d'água pequena. Essa tecnologia foi utilizada na explanação sobre tema. Por fim, ao terceiro momento, foi realizada uma dinâmica tipo quiz com grupos menores, verificando a sedimentação da aprendizagem. Após isso, foram distribuídos sanduíches naturais, como incentivo para a prática de hábitos de alimentação saudável.

Dentre os 18 participantes da ação educativa, havia 11 meninas (61\%) e 7 meninos (39\%), com faixa etária de 12 a 14 anos. O diálogo revelou que a maioria dos alunos não era praticante de qualquer atividade física. Também a maior parte deles comentou que rotineiramente não se alimentavam de maneira saudável; um número considerável alegou preferir e consumir bastante refrigerantes e menores volumes de suco e água. A totalidade de alunos referiu ter alguém na família com pelo menos um tipo de doença cardiovascular ou dislipidemias, destacando-se casos de diabetes, hipertensão arterial e infarto agudo do miocárdio.

A partir dessa experiência, observou-se como os fatores de risco modificáveis para doenças cardiovasculares estão radicados no atual estilo de vida de crianças e adolescentes, sobretudo por comporem naturalmente o cotidiano alimentar, familiar e social desses indivíduos. Pôde-se notar também como a aplicação de métodos diferentes dos usuais, ainda que no ambiente intraescolar tradicional, é providencial para se obter, de modo fluido, informações gerais dos indivíduos e sobre seus processos de saúde e doença ligados ao dia a dia, o que, se compreendeu serem peças importantes na elaboração de planos de cuidados voltados a grupos.

Para as estudantes de enfermagem a lida com grupos de crianças e adolescentes foi desafiadora, pois exemplificou diferenças, variedades de fenômenos e peculiaridades de cada participante, os quais se reconheceu que o enfermeiro igualmente encontra durante sua prática no cuidado coletivo. Esse perfil etário 
instigou a necessidade de aprimorar as habilidades para brevemente construir um vínculo de confiança com os participantes, manter a atenção e a colaboração deles durante a ação educativa e, principalmente, sensibiliza-los sobre o tema abordado, construindo o conhecimento de acordo com a realidade por eles vivenciada.

O desafio de trazer inovação ao ambiente de construção do conhecimento tornou-se base para as acadêmicas de enfermagem aperfeiçoarem a capacidade criativa, crítica e reflexiva e, nessa experiência, a elaboração de um instrumento facilitador foi efetivamente uma instrumentação exitosa para garantir aceitação do grupo alvo e ainda apreender a atenção, despertar a curiosidade e garantir a participação, itens estes indispensáveis em todas as etapas da educação em saúde aplicada, e assim propagar a informação.

Percebeu-se que o sucesso da ação educativa dependeu de fatores como: uso de elementos inovadores, como o recurso didático elaborado pelas autoras especificamente para reproduzir os conteúdos abordados; uso racional do tempo, ajustando as etapas da ação a um cronograma curto, porém, suficiente para construir o conhecimento, sem se tornar enfadonho, exaustivo ou deixar de ser atrativo aos participantes; uso de linguagem clara, acessível e atual, de acordo com a faixa etária, não vulgar, mas cotidiana, alinhada ao perfil dos participantes e; a opção por atividades em grupos, o que instigou a gregária e maior participação, construção de vínculo, este essencial para garantir a sedimentação e propagação do conhecimento fortalecido naquele momento.

\section{DISCUSSÃO}

Segundo Guedes NG, et al. (2019) atualmente o sedentarismo representa o hábito de vida de muitos adolescentes, e comportamentos sedentários como passar um grande período de tempo em frente a televisão, usar computadores, tablets e smartphone vem crescendo em meio a este grupo. Visto que a infância e a adolescência são períodos importantes no desenvolvimento de estilo de vida saudável para que este tenha continuidade ao longo da vida.

Sumini KL, et al. (2017) declara que está sendo rotineira as práticas alimentares inadequadas no período da infância e adolescência tais como, a substituição de refeições saudáveis, favorecendo a preferência por consumo de alimentos ricos em gorduras, açucares e lanches fest food, e que esta escolha por alimentos de baixo valor nutritivo tem gerado impacto negativo e contribuinte para o surgimento de distúrbios nutricionais como fator de risco para DCV precoce.

Em concordância, Moraes DM, et al. (2018) ressalta que o ambiente escolar é favorável para desenvolver hábitos alimentares irregulares presentes entre crianças e adolescentes, como o consumo excessivo de alimentos ultra processados com elevado teor energético e bebidas açucaradas, e diminuição do consumo de alimentos in natura. Pois é neste período que essas práticas são consolidadas, onde ocorrem modificações no consumo alimentar devido às alterações fisiológicas e a influência que o meio social exerce sobre os adolescentes.

Brito LMS, et al. (2016) destaca em seu estudo que a influência da família em relação aos hábitos de vida e a predisposição genética tem forte associação a fatores de risco de DCV em adolescentes, salientando que familiares que apresentam HAS, obesos, sedentários e tabagistas, exercem função crucial no comportamento de risco de crianças e adolescentes. Assim, as práticas irregulares já na primeira idade somam-se ao histórico familiar desfavorável, tornando os adolescentes vulneráveis e passiveis de desenvolverem doenças crônicas possivelmente evitáveis.

Conforme Rangel SRV, et al. (2015) devem ser desenvolvidos programas de educação para a saúde no ambiente escolar com o proposito de obter informações e difundir conhecimentos dos fatores de riscos, na tentativa de minimizar o sedentarismo e o surgimento de doenças crônicas não transmissíveis. Com isso, a aplicação de metodologias ativas na escola tem forte influência no dia a dia dos jovens, não apenas dentro do ambiente escolar, mas na vida diária, pois estas irão facilitar o esclarecimento para a mudança de hábitos dos estudantes, visto que pessoas que praticam atividades físicas regularmente na infância e adolescência adquirem mais chances de se tornarem adultos ativos. 
Tavares EAO, et al. (2018) afirma que o estudante de enfermagem por meio de vínculo com a comunidade, passa a ser um sujeito participativo e responsável pela busca do aprendizado e estreitamento de confiança com a população, que são desenvolvidas no decorrer da formação acadêmica pela sua aproximação com a sociedade, tendo a oportunidade de aperfeiçoar suas competências e servido como objeto de informação por meio de ações educativas, e assim, desenvolver o pensamento crítico-reflexivo e superando os desafios.

Para Lemos APS, et al. (2017) o uso de métodos ativos de ensino tem sido importante para o alcance da construção crítica e reflexiva dos alunos, incentivando a participação ativa dos estudantes tanto na sua formação quanto no cuidado holístico, de acordo com os princípios do sistema único de saúde (SUS). Estimulando a autonomia, criatividade e corresponsabilidade em sua formação, tornando-os profissionaiscidadãos empenhados no cuidado.

Bonfim ES, et al. (2016) aponta que as ações educativas proporcionam aos acadêmicos de enfermagem práticas e habilidades de intervir nas melhorias e transformações da sociedade, tornando-o um estimulo para prevenção, promoção e proteção através de ações que transmitam o conhecimento, pois o convívio com a mesma representa uma ferramenta enriquecedora para promover saúde em âmbito coletivo, colaborando para a formação profissional e cidadã.

\section{CONSIDERAÇÕES FINAIS}

Considerando a experiência prática vivenciada nesta ação educativa as autoras observaram em seus achados que os adolescentes adotaram atualmente um estilo de vida de acordo com seu dia a dia e estão vivendo com hábitos irregulares e comportamentos sedentários que compõem de forma natural a sua rotina social e familiar, o que pode ocasionar em DCV precoce. Dessa forma, a ação educativa se torna um importante mediador no processo de ensino-aprendizagem contribuindo de forma significativa para a mudança desse cenário e facilitando a assimilação dos conteúdos abordados para a formação acadêmica na área da saúde. O trabalho exposto teve limitações, como uma amostra pequena frente ao universo, além da dificuldade em alcançar a atenção dos jovens e sensibiliza-los quanto a prevenção de doenças crônico-degenerativas não transmissíveis.

\section{REFERÊNCIAS}

1. AUDI CAF, et al. Fatores de risco para doenças cardiovasculares em servidores de instituição prisional: estudo transversal. Epidemiologia e Serviços de Saúde, Brasília, 2016; 25(2): 301-310.

2. BONFIM ES, et al. Práticas educativas do enfermeiro no cotidiano na estratégia saúde da família. Revista Saúde e Desenvolvimento, 2016; 9(5): 38-52.

3. BRITO LMS, et al. Influência da educação em saúde da família no comportamento de risco em adolescentes. Arquivos de Ciências da Saúde, 2019; 23(2): 60-64.

4. FARIAS AM, et al. Fatores determinantes para prevenção de doenças cardiovasculares em adolescentes. Revista Interdisciplinar, 2016; 9(1): 34-40.

5. GUEDES NG, et al. Validação clínica dos fatores associados ao estilo de vida sedentário em adolescentes. Revista da Rede de Enfermagem do Nordeste, 2019; 20(e40395): 1-8.

6. FONTES VS, et al. Quemerina e fatores relacionados ao risco cardiovascular em crianças e adolescentes: Uma revisão sistemática. Revista Paulista de Pediatria, 2018; 36(2): 221-229.

7. LEMOS APS, et al. Percepção de estudantes de enfermagem sobre o portifólio como estratégia de ensino, aprendizagem e avaliação. Journal of Management \& Primary Health Care, 2017; 9(e7): 1-17.

8. LIMA KLL, et al. Ações educativas para promoção de saúde: prática de atividade física de estudantes do ensino médio. Revista Eletrônica do Curso de Pedagogia do Campus Jataí - UFG, 2015; 11(1): 1-18.

9. MORAES DM, et al. Exposição a mídia e hábitos alimentares de adolescentes em uma escola privada de São LuizMA. Revista de Investigação Biomédica, 2018; 10(2): 165-171.

10. ORGANIZAÇÃO PAN-AMERICANA DA SAÚDE - BRASIL (OPAS). Doenças cardiovasculares - principais fatos. Temas de Saúde, Brasília-DF, 2017; 4 p.

11. RANGEL SRV, et al. Atividade física e comportamento sedentário: prevalência e fatores associados em adolescentes de três escolas públicas de pelotas/RS. Revista Biomotriz, 2015; 9(01): 2317-3467. 
12. SANTOS EGR, et al. Prevalência de risco cardiovascular a partir de parâmetros antropométricos em crianças e adolescentes. Revista de Atenção à Saúde, 2019; 17(60): 54-62.

13. SUMINI KL, et al. Alimentação, risco cardiovascular e nível de atividade física em adolescentes. Revista Brasileira de Obesidade, Nutrição e Emagrecimento, 2017; 11(61): 23-30.

14. TAVARES EAO, et al. Intervenção comunitária na educação em enfermagem: relato de experiência. Revista Brasileira de Enfermagem, 2018; 71(suppl 4: 1878-1883.

15. VALE MEG, et al. Fatores de risco cardiovasculares e qualidade de vida em universitários. Revista de Enfermagem UFPE on line, 2018; 12(10): 2743-2752. 